\title{
Déni du travail et tyrannie des normes
}

Quand les normes de service deviennent une fin en soi à la SNCF

Denial of work and tyranny of norms. When norms of service become an ultimate finality at the SNCF

\section{Damien Collard}

\section{OpenEdition \\ Journals}

Édition électronique

URL : http://journals.openedition.org/travailemploi/5832

DOI : 10.4000/travailemploi.5832

ISSN : $1775-416 \mathrm{X}$

\section{Éditeur}

DARES - Ministère du Travail

\section{Édition imprimée}

Date de publication : 15 décembre 2012

Pagination : $35-48$

ISSN : 0224-4365

Référence électronique

Damien Collard, « Déni du travail et tyrannie des normes », Travail et Emploi [En ligne], 132 | octobredécembre 2012, mis en ligne le 01 décembre 2014, consulté le 30 avril 2019. URL : http:// journals.openedition.org/travailemploi/5832 ; DOI : 10.4000/travailemploi.5832 


\title{
Déni du travail et tyrannie des normes. Quand les normes de service deviennent une fin en soi à la SNCF
}

\author{
Damien Collard $(*)$
}

L'objet de cet article est d'explorer, dans une perspective clinique, les mécanismes organisationnels qui favorisent le déni du travail, à partir de l'exemple de deux démarches qualité déployées à la SNCF dans le domaine de la relation de service dans les années 2000, les projets Transilien et Gares en mouvement. Les normes de service définies dans le cadre de ces deux démarches ont contribué au déni du travail des agents en front office de la SNCF, essentiellement parce que le respect de ces normes est devenu une fin en soi. Cette situation a fait naître chez ces agents une activité "contrariée", telle qu'elle a été conceptualisée par Yves Clot, dans la mesure où ils ont perçu les normes comme une entrave à la réalisation d'un travail de qualité. Dans les deux cas étudiés, le déni a porté simultanément sur la réalité (dans laquelle le travail de ces agents devait s'exercer), le travail réel (par opposition au travail prescrit) et le "réel du travail» (au sens où l'entend la psychodynamique du travail, autrement dit ce qui résiste aux prescriptions organisationnelles).

Le déni du travail serait-il une tendance forte des organisations productives contemporaines comme l'affirment certains auteurs? Si tel est le cas, comment se manifeste-t-il et qu'entend-on au juste par «déni du travail»?

Pour Vincent de Gaulejac (2005), la prégnance d'une conception gestionnaire du travail favorise une méconnaissance des situations de travail réelles, le déni étant une des figures de cette méconnaissance (LHUiLIER, 2009). Selon Thomas PÉrilleux, certaines méthodes d'évaluation du travail contribuent au déni car elles ne prennent pas en compte le caractère multidimensionnel du travail et occultent le «travail vivant» (au sens de Marx) "en l'écrasant sous des mesures "indiscutables" et "exhaustives"» (Perilleux, 2005, p. 113). Dans la même veine, Amaury GrIMAND (2004) souligne que les techniques d'évaluation des compétences, qui sont mises en place dans les entreprises au nom de la «logique compétence», marquent un déni du travail réel quand elles ne prennent en compte que la seule performance (c'est-à-dire l'atteinte d'un résultat). Quant à Sophie Avarguez (2009), elle soutient que les prescriptions organisationnelles sont susceptibles d'alimenter le déni du travail réel et la souffrance au travail, son corollaire. «La souffrance mentale se prête [d'ailleurs] d'autant mieux au déni qu'elle a peu de signes visibles et qu'il en est souvent de même de ses causes » (Gollac et al., 2006, p. 39). Différentes formes de déni sont

(*) Laboratoire d'économie et de gestion (LEG) - Université de Bourgogne; damien.collard@univ-fcomte.fr ainsi mises en évidence par AvARguEz dans le cas des conseillers à l'emploi de l'Agence nationale pour l'emploi (ANPE). La dictature du chiffre et la prescription d'un idéal (DuJarier, 2004; GaUlejaC, 2005), l'empilement des instructions et l'injonction permanente au changement, ainsi que l'occultation du volet «social» de la relation de service, en constituent les principaux exemples.

Ces travaux envisagent le déni comme un refus de reconnaître une réalité. Cette conception concorde avec les définitions du déni données dans le champ de la psychiatrie, de la psychopathologie et de la psychanalyse, à savoir un mécanisme de défense à l'égard d'une perception dérangeante d'une réalité externe pour Sigmund FreUd (1909), une «tentative de désavouer, de renier l'existence d'une réalité déplaisante» (Grangaud, 2001, p. 11), ou encore «un phénomène de refus de prendre en compte une part de réalité externe inacceptable, c'est-à-dire non métabolisable sur le plan psychique» (BARDOU et al., 2006, p. 99).

Cependant, certains cliniciens du travail prennent soin de distinguer la «réalité» du «réel» dans leurs analyses (LhUiLIER, 2006). Cette distinction serait même au cœur de la clinique du travail. Ainsi, pour Christophe Dejours (1995), le fondateur de la psychodynamique du travail, tandis que la réalité est «un état de choses» (p. 42), le réel est «ce qui, dans le monde, se fait connaître par sa résistance à la maîtrise technique et à la connaissance scientifique » (p. 41). Selon lui, le réel se manifeste «sous la forme de l'expérience au sens d'expérience vécue» (p. 42), celle-ci étant toujours «une expérience subjective 
de l'échec, de l'incertitude, de l'impuissance, du doute» (Dejours, 2006, p. 128). Pour Dejours (1998, p. 30), le déni porte donc avant tout sur le «réel du travail», entendu au sens de "ce qui résiste aux connaissances, aux savoirs, aux savoir-faire et d'une façon plus générale à la maîtrise». Autrement dit, les prescriptions organisationnelles - c'est-à-dire les consignes, les procédures, les modes opératoires, ou encore les normes de qualité, etc. - sont inopérantes pour faire face au «réel du travail». Ce constat est partagé par d'autres auteurs, à commencer par Yves Clot et Dominique Lhuilier, pour qui «le monde du travail témoigne massivement d'un processus de réduction symbolique et de pratiques managériales de plus en plus déconnectées des réalités, tout entières au service d'une occultation du réel, de ce qui résiste aux savoirs, aux savoir-faire, à la technique, à la maîtrise» (LhUiLIER, 2009, p. 92). Yves Clot et Daniel FAÏTA (2000, p. 35) ajoutent que «le réalisé n'a pas le monopole du réel», dans la mesure où «le possible et l'impossible font partie du réel». Autrement dit, le réel doit être appréhendé non seulement sous l'angle de l'échec, mais aussi, et surtout, comme une occasion de développement, tout en sachant que ce développement peut être « contrarié» (Clot, 1995, 1999; Lhuilier, 2009).

Mais, au-delà de ces distinctions, quels sont les ressorts du déni du travail, ses fondements, et les mécanismes organisationnels qui le soutiennent?

L'objet de cet article est d'explorer, dans une perspective clinique, les mécanismes organisationnels qui favorisent le déni du travail pour remonter aux sources de ce déni, en partant de l'exemple de deux démarches qualité qui ont été déployées à la SNCF dans les années 2000, dans le domaine de la relation de service, et qui se sont traduites par la mise en place de normes de service prégnantes. Après avoir présenté le cadre organisationnel dans lequel ces deux démarches ont pris place, et notre méthodologie, nous étudierons les effets induits par ces démarches sur le travail des agents en front office de la SNCF, avant de discuter le bien-fondé de ces normes de service et d'expliquer pourquoi leur respect est devenu une fin en soi.

\section{Deux démarches qualité incarnant la "stratégie orientée client" de la SNCF}

Ces démarches constituent l'incarnation de la «stratégie orientée client» qui, adoptée par l'entreprise depuis la fin des années 1990 (Collard, 2010), est la résultante de trois évolutions importantes : le développement de la fonction commerciale dès la fin des années 1960 et la montée en puissance de la figure du «client» dans la stratégie de l'entreprise; la multiplication des démarches qualité et la diffusion dans l'organisation d'outils de gestion de la qualité; l'ouverture progressive de l'entreprise à la concurrence sous l'effet de la libéralisation du rail (voir tableau ci-dessous).

Tableau : Quelques grandes étapes de l'histoire de la SNCF et de la libéralisation du rail

\begin{tabular}{|c|c|}
\hline Dates-clés & Événements \\
\hline $\begin{array}{l}1 \text { er janvier } \\
1938\end{array}$ & $\begin{array}{l}\text { Création de la SNCF (Société nationale des } \\
\text { chemins de fer français). }\end{array}$ \\
\hline $\begin{array}{l}\text { Fin des } \\
\text { années } 1960 \\
\text { - années } 1970\end{array}$ & $\begin{array}{l}\text { Émergence de la fonction commerciale } \\
\text { (WILLIOT, 2005) : lancement des études } \\
\text { commerciales (1967), mise en place d'une } \\
\text { politique de tarification pour ajuster l'offre à } \\
\text { la demande, etc. }\end{array}$ \\
\hline $1972-1973$ & $\begin{array}{l}\text { Réforme des structures de l'entreprise, avec } \\
\text { notamment la création de vingt-cinq régions } \\
\text { pilotées par des directeurs de région. }\end{array}$ \\
\hline $\begin{array}{l}\text { Fin des } \\
\text { années } 1980 \\
\text { - début des } \\
\text { années } 1990\end{array}$ & $\begin{array}{l}\text { «L'orientation client» devient une règle de } \\
\text { base du management (GUÉLAUD, 2002) et } \\
\text { inspire certaines réformes : décentralisation } \\
\text { des décisions, concentration des établisse- } \\
\text { ments, contractualisation interne (CAUCHON, } \\
\text { 1998). }\end{array}$ \\
\hline 1996 & $\begin{array}{l}\text { Création d'une Direction du développement } \\
\text { des gares et émergence de services en gare : } \\
\text { services d'information, de portage, agents } \\
\text { d'ambiance, etc. (PouvelLE, 1998). }\end{array}$ \\
\hline $\begin{array}{l}13 \text { février } \\
1997\end{array}$ & $\begin{array}{l}\text { Création de Réseau ferré de France (RFF), } \\
\text { chargé de la gestion des infrastructures et } \\
\text { propriétaire du réseau, dans le cadre de } \\
\text { l'ouverture à la concurrence (en application } \\
\text { de la directive européenne 91-440). }\end{array}$ \\
\hline $\begin{array}{l}\text { Fin des } \\
\text { années } 1990\end{array}$ & $\begin{array}{l}\text { Adoption d'une «stratégie orientée } \\
\text { client», via le déploiement de la «gestion } \\
\text { par activité»», puis du projet phare «Cap } \\
\text { Client». }\end{array}$ \\
\hline Fin 1999 & $\begin{array}{l}\text { Lancement du Transilien et mise en place } \\
\text { de l'humanisation des gares et des trains en } \\
\text { Île-de-France. }\end{array}$ \\
\hline 2002 & $\begin{array}{l}\text { Organisation de certaines gares selon un } \\
\text { concept qui privilégie le regard du voya- } \\
\text { geur: «l'Escale» (KOPECKY, 2002); la } \\
\text { Direction du développement des gares } \\
\text { devient la Direction des gares et de l'escale. }\end{array}$ \\
\hline 2003 & $\begin{array}{l}\text { Lancement du programme Gares en mouve- } \\
\text { ment (démarche qualité) en vue d'accélérer } \\
\text { le processus de certification des services en } \\
\text { gare (FAVIN-LÉVĖQUE, 2005). }\end{array}$ \\
\hline 2003-2004 & $\begin{array}{l}\text { Généralisation de l'Escale dans les gares et } \\
\text { définition de quarante-cinq engagements en } \\
\text { matière de qualité de service (ROYER et al., } \\
\text { 2004). }\end{array}$ \\
\hline 7 avril 2009 & $\begin{array}{l}\text { Création d'une nouvelle branche pour } \\
\text { préparer l'ouverture à la concurrence } \\
\text { du transport de voyageurs : Gares et } \\
\text { Connexions. }\end{array}$ \\
\hline $\begin{array}{l}1^{\text {er janvier }} \\
2010\end{array}$ & $\begin{array}{l}\text { Ouverture à la concurrence du transport } \\
\text { international de passagers. }\end{array}$ \\
\hline
\end{tabular}

La SNCF est ainsi passée progressivement d'une organisation par métiers à une structure par types de client, avec des branches positionnées sur des segments de clientèle (correspondant aux différents donneurs d'ordre de l'entreprise) et des directions au service des branches couvrant les métiers ferroviaires traditionnels (directions métier) ou des activités spécifiques (directions d'activité). 
La première démarche étudiée - le lancement du Transilien et de l'humanisation des gares et des trains en Île-de-France à la fin de l'année 1999 - témoigne de la volonté de la Direction générale de la SNCF de transférer certaines attributions des directions régionales parisiennes ${ }^{(1)}$ vers la Direction Île-de-France (2) afin de lui permettre de répondre aux demandes de ses clients : les usagers des trains de banlieue (destinataires du service) et le Syndicat des transports en Île-deFrance (client institutionnel). La Direction Île-de-France a ainsi progressivement repris un certain nombre de dossiers auparavant gérés par ces directions régionales : l'évolution des métiers commerciaux, le pilotage du programme gouvernemental «Nouveaux services - Emplois jeunes » en Île-de-France et la redéfinition du rôle des agents d'ambiance embauchés sous statut emploijeune par la $\mathrm{SNCF}^{(3)}$, l'émergence de nouveaux services dans les gares, la mise en place de standards de service, etc. Pour ce faire, elle a édicté des directives très précises qui ont dû être respectées scrupuleusement par les directions régionales et, à une échelle locale, par les unités opérationnelles concernées.

Figure 1 : La démarche Transilien

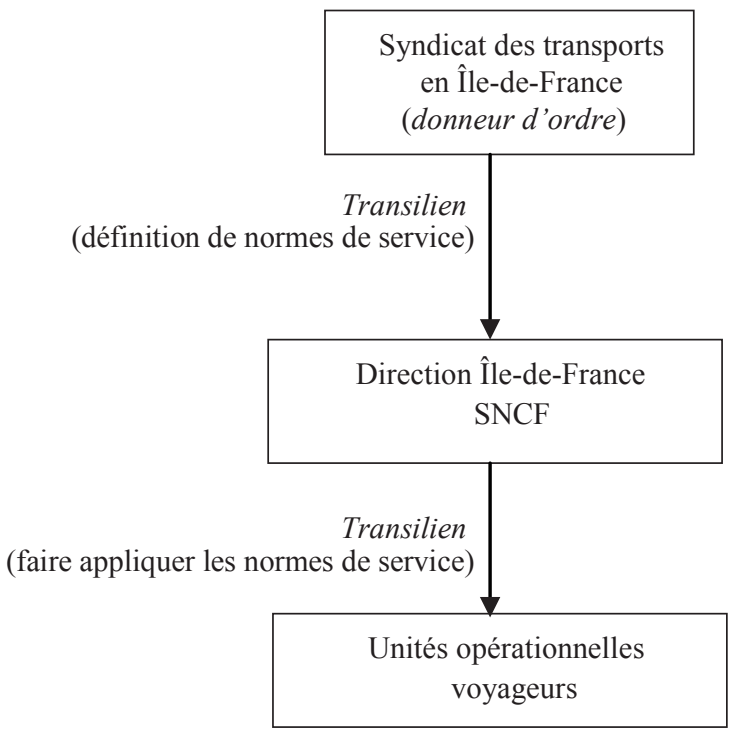

(1) Il s'agit des directions régionales de Paris Sud-Est, Paris Rive-Gauche, Paris Est et Paris Nord.

(2) La Direction Île-de-France est une direction d'activité.

(3) Le programme «Nouveaux services - Emplois jeunes» avait été lancé par le gouvernement de Lionel Jospin en 1998.

Dans le cadre de ce programme, environ 2000 jeunes avaient été recrutés comme contractuels par la SNCF, essentiellement sur des fonctions d'agent d'ambiance. Les tâches qui avaient été confiées à ces jeunes relevaient essentiellement de l'accueil et de l'accompagnement des voyageurs dans les gares ainsi que de la médiation sociale.
La seconde démarche - baptisée «Gares en mouvement», lancée durant l'année 2003 - constitue, quant à elle, un des volets principaux de la politique commerciale définie par la Direction des gares et de l'escale ${ }^{(4)}$ et incarne la volonté de la SNCF de mieux prendre en compte les besoins des voyageurs, usagers des TER et des trains grandes lignes. Elle témoigne également de la mainmise de cette direction sur les services d'escale qui offrent aux clients des prestations en matière d'information, d'accueil, d'orientation, etc., via notamment la diffusion auprès des agents d'escale d' «attitudes de service».

Ces deux démarches qualité ont impliqué une contractualisation entre les directions d'activité et leurs donneurs d'ordre, l'introduction de relations de type client-fournisseur en interne, et la définition de normes de service (voir figures 1 et 2 ci-dessous).

Dans les deux cas, nous avons observé et analysé (voir encadré 1) les effets induits par ces démarches qualité sur le personnel en front office - agents d'ambiance et agents commerciaux dans un cas, agents d'escale dans l'autre - en étant particulièrement attentif, dans une perspective clinique, au sens que ce personnel donnait à ces évolutions et à leur vécu au travail(5).

Figure 2 : La démarche Gares en mouvement

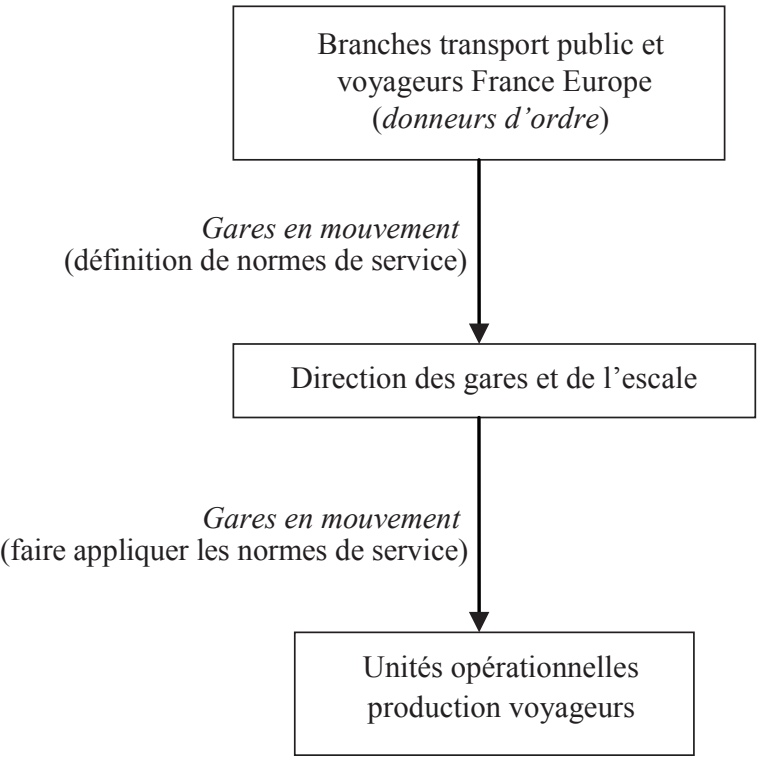

(4) La Direction des gares et de l'escale est une direction d'activité.

(5) Dans les deux cas, il s'agit d'employés de service situés en bas de la hiérarchie à la fois sur le plan statutaire et en termes de rémunération. Cependant, la situation des agents commerciaux et des agents d'escale doit être soigneusement distinguée de celle des agents d'ambiance recrutés par la SNCF en tant qu' "emplois-jeunes». Tandis que les premiers étaient des agents statutaires et bénéficiaient à ce titre de tous les avantages liés au statut de cheminot, exerçant un métier reconnu par l'entreprise, les seconds étaient des agents contractuels - donc au statut précaire - exerçant un «métier» en émergence et par conséquent non reconnu institutionnellement. 


\section{Encadré 1 \\ Méthodologie de la recherche}

Les conséquences du lancement du Transilien et de la mise en place de l'humanisation des gares ont été étudiées dans le cadre d'une thèse de doctorat effectuée sous convention CIFRE (convention industrielle de formation par la recherche) au sein de la SNCF (ColLARD, 2002). Intégré en tant que "chargé de mission" au sein de la Direction régionale des ressources humaines de Paris Sud-Est, nous avons endossé différents rôles. Nous avons tout d'abord analysé de près le travail réalisé par les équipes d'agents d'ambiance travaillant dans les gares rattachées à l'Établissement d'exploitation de Melun (dénommé «Établissement de Melun " dans la suite de cet article) ${ }^{(1)}$. Pour ce faire, nous avons notamment procédé par observation participante sur l'une des gares les plus «sensibles» de l'Établissement de Melun - celle d'Évry-Courcouronnes - en intégrant l'équipe des agents d'ambiance pendant cinq semaines en 1999. Nous avons également animé un groupe de travail intitulé "veille professionnelle et enrichissement des tâches " en 1999 et 2000, composé d'encadrants d'équipes d'agents d'ambiance de l'Établissement de Melun, dont la mission était de réfléchir au contenu des tâches confiées aux agents d'ambiance en vue de les enrichir. Par ailleurs, nous avons conduit une étude sur l'impact économique de la présence des équipes d'agents d'ambiance dans les gares rattachées à cet Établissement dans le but d'évaluer la contribution de leur travail à la sûreté des gares.

La démarche Gares en mouvement et les effets de la mise en place des «attitudes de service " au sein des services d'escale ont été étudiés lors d'une recherche collective menée pour le compte de la SNCF en 2007 (BoRzEIX et al., 2008). À cette occasion, nous avons constaté la prégnance des «attitudes de service" dans les pratiques de gestion des ressources humaines et dans les discours des agents d'escale. Nous prendrons l'exemple d'un des services d'escale étudié pour illustrer notre propos. Pour analyser l'activité des agents d'escale de ce service-agents d'accueil et adjoints départ de train-, nous avons mené quatre entretiens semi-directifs avec l'encadrement intermédiaire ${ }^{(2)}$ et trois avec des agents d'accueil. En outre, des entretiens en situation de travail avec le chef d'escale, avec plusieurs adjoints départ de train sur les quais, et avec tous les agents d'accueil sur les quais et dans la bulle d'accueil ont été réalisés. Des entretiens avec des représentants de la Direction des gares et de l'escale ont également été menés et plusieurs réunions de restitution des résultats de la recherche ont été organisées. Par ailleurs, une observation fine de l'activité des agents d'accueil et des adjoints départ de train a été effectuée(3). Enfin, une analyse documentaire approfondie a été conduite sur la base des traces existantes : fiches de postes, référentiels de compétences, notes de service, guides des «bonnes pratiques», etc.

(1) À noter que la Direction régionale de Paris Sud-Est avait fait le choix de mettre en place des équipes d'agents d'ambiance pilotées par des «encadrants», c'est-à-dire des cheminots volontaires pour encadrer une équipe de jeunes et structurer des activités naissantes dans le champ de la médiation sociale et de l'accueil en gare. La région de Paris Sud-Est était l'une des rares régions à avoir adopté ce mode d'organisation.

(2) Un avec le dirigeant de l'unité opérationnelle production voyageurs, trois avec le dirigeant de proximité Escale.

(3) Cette observation s'est étalée sur sept journées et a représenté vingt-huit heures de présence sur le terrain aux côtés des agents, à différents moments de la journée, en période creuse et en période de pointe. À cette occasion, des dizaines d'interactions entre agents et clients furent enregistrées et retranscrites dans un journal de bord, de même que les nombreux commentaires faits a posteriori par les agents.

\section{L'humanisation des gares : une démarche vide de sens}

La démarche Transilien, lancée fin 1999, consistait à labelliser les gares et les trains qui répondaient à certains standards de qualité. Concernant les gares, les engagements de la SNCF vis-à-vis de son donneur d'ordre, le Syndicat des transports en Île-deFrance, portaient principalement sur l'humanisation des gares, dispositif qui devait impérativement se traduire par la présence d'au moins deux agents de la SNCF par gare jusqu'au passage du dernier train. Il s'agissait également pour la SNCF de réhabiliter les gares en renforçant la signalétique, en remettant à neuf les peintures et en remplaçant les bancs vétustes notamment. Elle s'engageait également à maintenir en bon état de marche les différentes installations comme les escalators, à assurer la propreté et la création d'une ambiance agréable par la diffusion de musique, la décoration florale, etc. Un système de bonus-malus, des procédures de contrôle et des indicateurs de qualité avaient d'ailleurs été mis en place pour l'inciter à respecter ses engagements.

Si la norme de service était claire et sans ambiguïté dans le cas de l'humanisation des gares puisqu'il s'agissait de garantir au client la présence en gare d'un collectif de taille réduite composé obligatoirement d'un agent commercial (6), d'un agent d'ambiance et éventuellement d'un maître chien sur les gares les plus «sensibles» jusqu'au passage du dernier train, tel ne fut pas le cas pour le contenu du travail demandé aux agents puisqu'aucune réflexion sur leurs missions et tâches concrètes ne fut conduite par la Direction Île-de-France. La seule obligation des agents était en définitive d'assurer

(6) Agent de guichet, chargé de la vente de billets, ou agent d'accueil, itinérant en gare. 
une présence continue dans le hall de la gare et/ou sur les quais notamment lors de l'arrivée des trains en gare. C'est donc un déficit de prescription qui a caractérisé la mise en œuvre de l'humanisation des gares. Cette situation, qui s'est avérée dans les faits subjectivement très coûteuse pour les agents ${ }^{(7)}$, est d'autant plus paradoxale que l'un des projets de la Direction Île-de-France était d'assurer un transfert croisé de compétences entre agents commerciaux et agents d'ambiance : tandis que les premiers devaient transmettre aux seconds leurs connaissances du monde ferroviaire, les seconds devaient apporter aux premiers leur expérience des codes sociaux et culturels en vigueur dans les cités "sensibles» ${ }^{(8)}$. Ce déficit de prescription, et l'absence de mesure de soutien et d'accompagnement, eurent pour effet de vider de son sens la démarche d'humanisation. Ainsi, les agents d'ambiance appréhendèrent leur nouveau rôle comme celui d'un "gardien de gare», impuissant à agir en cas de problème. L'humanisation des gares leur apparut, au mieux comme un dispositif inadapté à la nature des problèmes rencontrés dans les gares, au pire comme une démarche vide de sens, et, dans tous les cas comme un facteur de démotivation.

Pourtant, les agents d'ambiance n'étaient $a$ priori pas hostiles au principe de l'humanisation qui consistait à assurer une présence la nuit pour accueillir et sécuriser les voyageurs. Mais en comparaison du travail de médiation réalisé auparavant au sein des équipes d'agents d'ambiance, un travail à leurs yeux consistant, motivant et gage d'efficacité (voir encadré 2), ils évaluèrent négativement leur nouveau rôle et le contexte dans lequel ils l'exerçaient : un collectif hétérogène de taille restreinte réduit le plus souvent à l'impuissance...
(7) Pour CLOT, le relâchement de la prescription et l'atrophie de la tâche qui s'ensuit (situation que l'on rencontre fréquemment dans les services) ont pour effet de priver les salariés des appuis organisationnels nécessaires à l'accomplissement d'un travail de qualité (Сцот, 2010, p. 66)

(8) À noter que la plupart des agents d'ambiance embauchés par l'Établissement de Melun avaient été recrutés selon des critères sociaux, ethniques et sexuels (BorzeIx, Collard, 1999). Emmanuelle LADA (2003), de son côté, avait constaté en région parisienne une "hégémonie masculine» dans le cas des équipes d'agents d'ambiance de la SNCF. Par ailleurs, le partage d'un même habitus avec les jeunes issus des cités était censé favoriser une certaine "connivence» avec ces jeunes (Petitclerc, 2002) et faciliter l'intervention des «médiateurs» auprès de ce public (DivaY, 2004).

\section{Encadré 2}

\section{Les compétences des agents d'ambiance de la gare d'Évry-Courcouronnes (en 1999)}

L'observation participante que nous avions menée au sein de l'équipe des agents d'ambiance de la gare d'Évry-Courcouronnes dans le courant de l'année 1999 avait révélé la présence de compétences qui étaient à la fois individuelles et collectives. Elles reposaient sur des savoirs et des savoirfaire qui étaient partagés et/ou distribués entre les membres de l'équipe (ColLARD, 2003).

Nous avions ainsi constaté que les agents d'ambiance de la gare d'Évry-Courcouronnes catégorisaient de manière extrêmement fine les différents publics. Par exemple, parmi les «indésirables» de la SNCF, les agents distinguaient les «zonards", les «toxicos", les "psychos", les «mythos", les «hystériques», les «mystiques", etc. Cette terminologie, qui leur était propre, équivalait à des mini-schèmes d'action. Elle permettait une économie d'interprétation et l'adoption de comportements-types pertinents : être détendu ou vigilant, créer de la convivialité ou garder ses distances, plaisanter ou rester froid et laconique, dialoguer ou faire un «coup de pression ", etc.

Cependant, tous les agents d'ambiance n'avaient pas développé les mêmes compétences. Nous avions en effet remarqué qu'ils s'étaient implicitement répartis les rôles en fonction des ressources qui étaient les leurs et du type de problème rencontré. Ainsi, certains agents issus des cités "sensibles" s'étaient spécialisés dans la gestion des relations avec les «indésirables», tandis que d'autres prenaient plus volontiers en charge les tâches relatives à l'information et à l'orientation des voyageurs.

Les échanges que nous avons eus à cette époque avec les agents lors de nos visites sur site, mais également l'analyse de certaines traces écrites (mains courantes, comptes rendus de réunions, etc.), ont révélé deux phénomènes : un sentiment d'inutilité ressenti par les agents d'ambiance, du fait que leur travail se cantonnait à assurer une présence en gare alors même que la fréquentation était très réduite en grande soirée (d'où le terme péjoratif de «gardien de gare» utilisé par les agents pour décrire leur rôle dans le cadre de l'humanisation $\left.{ }^{(9)}\right)$; des tensions au sein même des équipes d'humanisation lors de situations conflictuelles avec des «indésirables» de la SNCF (toxicomanes, sans domicile fixe $[\mathrm{SDF}]$, etc.), tensions générées, d'après les agents, par la présence et l'attitude des maîtres chien

(9) En plus d'assurer une présence en gare, le comptage des clients au-delà de minuit a été la seule «véritable» mission qui leur a été confiée. 
(qualifiées de «sécuritaires» $\left.{ }^{(10)}\right)$ et par l'impossibilité dans laquelle ils se trouvaient de poursuivre le travail de médiation sociale entrepris auparavant dans le cadre des équipes d'agents d'ambiance.

Un agent d'ambiance, évoquant l'humanisation des gares explique ainsi : "De toute façon la médiation ne peut pas être faite à ces heures-là!» Un autre agent ajoute : "Le métier d'agent d'ambiance c'est un travail d'équipe. Nous finalement notre métier ce $n$ 'est pas de travailler avec un agent du guichet qui lui n'a jamais fait de médiation! Et puis en plus là on est seul... Cela ne ressemble plus à notre travail.»

Coupés de leur équipe d'appartenance, de leur cadre habituel de travail et de leurs repères, ils n'ont pas pu mobiliser leurs compétences de médiation. La désillusion et la frustration qui en ont résulté sont révélatrices d'une activité «empêchée», «suspendue», ou encore «contrariée», pour reprendre la terminologie de CLOT (1999) (11), activité dont on sait qu'elle a un coût subjectif élevé. Cette démotivation collective fut d'ailleurs relayée par certains membres de l'encadrement de la Région de Paris Sud-Est.

Un membre de la Délégation régionale sûreté de Paris Sud-Est, précise ainsi dans un compte-rendu de réunion : «Une majorité d'emplois-jeunes vivent la dispersion des équipes formées depuis 1998 comme un retour en arrière sur le plan de la sûreté sur le réseau Île-de-France. D'après eux, leur mission a évolué d'un travail de prévention, de négociation avec les jeunes à problèmes dans les trains ou dans les gares vers une mission de présence passive sans marge de manouvre et sans possibilité de réaction en cas de problème.»

Un cadre de l'Établissement de Melun, évoquant la mise en place de l'humanisation des gares explique : «Depuis quelques semaines, des bandes de jeunes semblent apprécier nos fermetures tardives, en particulier à Savigny et à Boussy-SaintAntoine [...]. À partir de minuit, lorsque l'équipe d'emplois-jeunes et son encadrant ont terminé leur service et qu'il ne reste que deux agents et le maîtrechien dans la gare, la situation devient plus critique. Nous n'avons rencontré aucun agent réellement satisfait de son nouveau poste de "gardien de gare". Il est plus facile d'humaniser "sur le papier" que sur le terrain!»

Par ailleurs, le déficit de prescription à l'origine de l'humanisation des gares engendra, chez les agents

(10) Plusieurs altercations ont eu lieu entre des maîtres chien et des jeunes issus des cités. Après enquête, certains maîtres chien ont d'ailleurs été licenciés.

(11) Cette situation est révélatrice du «réel de l'activité», de ce qui aurait pu advenir, c'est-à-dire de ce que les agents auraient aimé pouvoir faire dans d'autres circonstances, en l'occurrence si l'occasion leur avait été donnée d'assurer l'humanisation des gares dans le cadre des équipes d'agents d'ambiance existantes. Incontestablement, cette «amputation du pouvoir d'agir» (СLOT, 1999) a été une source de souffrance. commerciaux, une montée de l'anxiété en raison de l'absence de tout cadre protecteur face aux phénomènes d'insécurité. Un tel cadre aurait pourtant facilité l'émergence de représentations et de pratiques partagées entre agents issus de différents métiers. Les agents commerciaux n'ont donc guère mieux vécu la situation que les agents d'ambiance, d'autant plus, d'une part, qu'ils ne furent pas en mesure de mobiliser leurs compétences commerciales car les demandes des clients en grande soirée sont quasiment inexistantes et, d'autre part, qu'ils n'avaient pas été formés pour gérer des situations délicates et potentiellement conflictuelles avec les «indésirables » ${ }^{(12)}$.

$\mathrm{Au}$ final, ce déficit de prescription, associé à une méconnaissance par la Direction Île-de-France de la réalité sociale des gares de banlieue au-delà d'une certaine heure, a contribué au déni du travail des agents en front office de la part de l'entreprise. Par ailleurs, la mise en place de roulements au sein des équipes en charge de l'humanisation eut pour conséquence de les fragiliser considérablement. Leur taille en fut mécaniquement réduite, d'autant plus que les agents devaient mettre en œuvre à la fois l'humanisation des gares et celle des trains en grande soirée en assurant non seulement une présence dans toutes les gares du périmètre mais aussi dans tous les trains jusqu'au dernier. Il en résulta des tensions au sein des équipes d'agents d'ambiance entre ceux qui s'étaient engagés dans la démarche d'humanisation pour des raisons financières et ceux qui s'y étaient refusés, une perte de l'esprit d'innovation, et un effritement des compétences individuelles et collectives ${ }^{(13)}$. La mise en place de l'humanisation a ainsi constitué un préambule à la disparition des équipes d'agents d'ambiance mises en place quelques années auparavant sur la Région de Paris Sud-Est.

Cette situation nous sembla d'autant plus dommageable que l'étude que nous avions entreprise sur l'impact économique de la présence des équipes d'agents d'ambiance dans les gares de l'Établissement de Melun avait révélé que celle-ci avait permis de faire diminuer de manière significative le nombre d'actes de malveillance sur la période 1998-2000 (14).

(12) Un rapport d'expertise, commandité par le comité d'hygiène, de sécurité et des conditions de travail (CHSCT) de l'Établissement de Melun et rédigé par un ancien médecin du travail de la SNCF, avait d'ailleurs mis en lumière le désarroi des agents commerciaux et les effets délétères de cette situation sur leur santé mentale.

(13) Il faut signaler que ces compétences étaient fragiles car elles dépendaient à la fois des individus qui les mettaient en œuvre, du degré de confiance et de coopération qui existait entre les membres d'une même équipe, et du contexte particulier dans lequel elles se déployaient (COLLARD, 2001).

(14) Nous avions en effet observé une baisse du nombre des actes de malveillance, sur la période considérée, dans les gares dotées de collectifs d'agents d'ambiance et sur la tranche horaire de présence des agents (16h-24h), alors que dans le même temps les actes de malveillance étaient tendanciellement en augmentation sur la tranche horaire $24 \mathrm{~h}-16 \mathrm{~h}$ et dans les gares où la présence d'agents d'ambiance était nulle ou réduite (deux ou trois agents au plus par gare). 
Elle tendait ainsi à corroborer les choix organisationnels de la Direction régionale de Paris Sud-Est et le bien-fondé des pratiques de médiation sociale. Pour autant, les compétences de médiation développées par les agents d'ambiance firent l'objet d'un véritable déni de la part de la Direction Ile-de-France. Le refus catégorique de ses responsables d'employer le terme de «médiation» pour qualifier l'activité des agents d'ambiance fut d'ailleurs, de notre point de vue, un symptôme de ce déni. Dans la mesure où l'un de leurs objectifs principaux était de «fondre» les emplois-jeunes dans la politique commerciale de la Direction Île-de-France et de recomposer les métiers commerciaux pour créer un métier unique d'agent commercial, résultat de la fusion des emplois d'agent de guichet, d'agent d'accueil et d'agent d'ambiance, accepter de parler de «médiation» revenait à prendre le risque de voir émerger un nouveau métier - agent d'ambiance - qui serait venu contredire les orientations stratégiques de cette direction et bousculer les catégories existantes à la SNCF. Les cloisonnements existants entre les filières professionnelles auraient été remis en cause, notamment entre la filière commerciale et la filière sûreté. À cheval entre le commercial et la sûreté, la «médiation» apparut donc comme un concept subversif (COLLARD, 2002).

L'histoire du lancement de l'humanisation des gares sur la Région de Paris Sud-Est est donc celle d'un double déni de la part de la Direction Île-deFrance : d'une part, déni du travail collectif et des compétences de médiation des équipes d'agents d'ambiance de l'Établissement de Melun en raison de la sous-évaluation de leur contribution au maintien de la sûreté dans les gares; d'autre part, déni du travail des agents engagés dans la démarche d'humanisation dû au déficit de prescription évoqué précédemment et à la méconnaissance, par la Direction Île-de-France, de la réalité sociale des gares de banlieue. Ce qui a été dénié, au final, fut à la fois la réalité du travail (à savoir le contexte social spécifique dans lequel les agents devaient intervenir), le «réel du travail» (matérialisé par les difficultés, les contraintes et les obstacles de toutes sortes rencontrés par les agents dans l'exercice de leur activité), et le travail réel (c'est-à-dire les compétences mobilisées par les agents d'ambiance, mais aussi le fait d'assurer une présence en gare, sans réel moyen d'action en cas de problème, pour ceux chargés de la mise en œuvre de l'humanisation des gares) ${ }^{(15)}$.

(15) Une différence de taille est cependant à noter. Tandis que dans le cadre des équipes d'agents d'ambiance, les agents ont réellement pu apporter des réponses pertinentes aux problèmes rencontrés en gare et, ce faisant, développer leur pouvoir d'agir, ils n'ont pas été en mesure de mobiliser leurs compétences de médiation dans le cadre de l'humanisation des gares et ont, de ce fait, été réduits à l'impuissance. Autrement dit, si dans un cas le travail réel est venu à bout du «réel du travail», dans l'autre, les agents ont buté sur ce réel.

\section{Les "attitudes de service" de Gares en mouvement : des normes idéales de la relation de service qui sont venues compliquer l'exercice du métier}

La seconde démarche étudiée dans cet article («Gares en mouvement») donna lieu à une contractualisation entre la Direction des gares et de l'escale et deux donneurs d'ordre internes à la SNCF, la branche voyageurs France Europe (grandes lignes) et la branche transport public (TER). Elle se concrétisa par la définition de normes de service que devaient respecter scrupuleusement les unités opérationnelles et le personnel d'escale, parmi lesquelles les «attitudes de service» qui consistaient en un savant mélange de normes vestimentaires, de scripts langagiers et de postures relationnelles à respecter dans toute relation de service (voir encadré 3).

\section{Encadré 3 \\ Les “Attitudes de service" à l'Escale (en 2007)}

Un document conçu par la Direction des gares et de l'escale à destination des agents d'escale au contact des voyageurs précisait que «les Attitudes de service décrivent notre manière d'être face à nos clients. Nous devons les adopter si nous souhaitons être reconnus comme de véritables professionnels du Service. Ces Attitudes sont écrites pour répondre au mieux aux attentes de nos clients. [...] Chaque client doit être accueilli avec la bonne Attitude, par tous les agents, quel que soit leur métier».

Ces attitudes étaient réparties en trois catégories - "attitude générale», "visibilité pour les clients ", « information donnée » - qui apparaissaient comme autant de «promesses» faites au client.

- La catégorie "attitude générale» recouvrait les attitudes suivantes : l'agent salue en premier lieu le client ou répond immédiatement à son salut par un "Bonjour, Monsieur» ou un «Bonjour, Madame»; est aimable et souriant tout au long du dialogue; est disponible et prend le client en considération; ose aller au-devant du client (il est proactif); prend poliment congé du client et accompagne la poursuite de son voyage.

- La catégorie «visibilité pour les clients» englobait : le port de la tenue complète; le port de chaussures de ville (en harmonie avec le reste de la tenue); le port d'une tenue propre et soignée; le port du badge avec son prénom et la mention «à votre service".

- À la catégorie «information donnée» correspondaient les attitudes suivantes : l'écoute active; renseigner de manière exacte et précise le client; expliquer tous les services avec pédagogie; disposer d'un espace de travail rangé et propre. 


\section{Encadré 4}

\section{Des outils de gestion entièrement focalisés sur les "attitudes de service"}

- Les comptes rendus de visite des enquêteurs client mystère (qui servaient de base pour évaluer la qualité des services rendus par le personnel d'escale) étaient rédigés à partir d'un support contenant six grandes catégories (les "attitudes de service" étaient l'une de ces catégories) qui se décomposaient elles-mêmes en différents items (les "attitudes de service" se décomposaient ainsi en vingtquatre items).

- Les documents de synthèse des enquêtes client mystère, élaborés par la société prestataire, présentaient les résultats consolidés des différentes enquêtes client mystère sous forme de graphiques (tous les trimestres) : à chaque grande catégorie correspondait une note globale exprimée en pourcentage qui matérialisait le degré de conformité des comportements des agents d'escale aux "attitudes de service ". Ils étaient systématiquement communiqués aux dirigeants de la SNCF.

- Le support d'évaluation confié au dirigeant de proximité Escale (conçu par la Direction des gares et de l'escale pour lui permettre d'évaluer ses agents) avait été élaboré sur la base du support utilisé par les enquêteurs de la société prestataire : il reprenait donc les catégories et items évoqués plus haut.

- La fiche d'évaluation "tandem» du dirigeant de proximité Escale (la première rubrique de cette fiche concernait les "attitudes de service») était utilisée dans le cadre des «tandems" (pratique d'évaluation qui consistait pour un encadrant de proximité à suivre un agent dans son travail en vue d'évaluer son comportement et ses compétences).

- Le livret de suivi professionnel reprenait systématiquement les évaluations formalisées dans le cadre des "tandems", dont celles relatives aux «attitudes de service».

- Dans les fiches de poste des agents d'escale figuraient également en bonne place les "attitudes de service » (elles faisaient donc partie intégrante du métier).

- Enfin, les «mémos service» établis par la Direction des gares et de l'escale permettaient de diffuser de manière pédagogique les "attitudes de service " auprès des agents d'escale.

La prise en compte et l'intériorisation de ces attitudes par les agents étaient régulièrement vérifiées lors d'enquêtes client mystère menées par une société travaillant pour le compte de la SNCF (appelée «société prestataire» dans la suite de cet article). Les résultats de ces enquêtes étaient censés refléter le point de vue du «client» et permettaient d'évaluer la conformité du service rendu à des standards préalablement définis. Les attitudes des agents étaient ainsi jugées «conformes» ou «non conformes» aux normes en vigueur. Prenons l'exemple de l'item «Les agents d'escale accueillent tous spontanément tout client qui s'adresse à eux par un "Bonjour, Monsieur" ou "Bonjour, Madame"» : si le client mystère avait croisé trois agents d'escale et si les trois avaient respecté le script, la réponse cochée était «conforme»; en revanche, elle était «non conforme» si un seul d'entre eux n'avait pas respecté le script. Cette «sur-prescription» de l'activité des agents d'escale contraste donc fortement avec la situation décrite précédemment. Les agents d'escale ont en définitive été pris dans un filet aux mailles extrêmement serrées. Ceci est d'autant plus vrai que tous les outils de contrôle et d'évaluation mis en place par la Direction des gares et de l'escale - des comptes rendus de visite des enquêteurs client mystère aux «mémos services» en passant par le livret professionnel de suivi de l'agent d'escale (voir encadré 4) - étaient centrés sur les normes de service.

Ces instruments constituaient des forces de rappel très puissantes pour les agents. Par ailleurs, ils renforçaient de facto le pouvoir des managers de proximité et leur permettaient d'asseoir leur autorité hiérarchique. L'encadrement intermédiaire pouvait ainsi déployer différentes tactiques pour discipliner les comportements (faire preuve de pédagogie, rappeler la règle, donner un ordre, faire une remontrance, etc.) et recourir à toute une palette de mesures, qui allait du blâme à la «sanction normalisatrice», pour reprendre l'expression de Michel FouCAult ${ }^{(16)}$ (1975) :

«Alors là où j'ai essayé d'avoir une action ce sont les nouvelles normes justement. Parce que les anciennes, elles sont à peu près connues. [...] Si je vois un agent avec des chaussures non conformes je lui fais la remarque. Je peux lui mettre un objectif dans son entretien annuel... Ce qu'il faut voir aussi c'est que la norme, à un moment donné, elle se met en place. On lui fait la remarque, c'est le management. Et puis, à un moment donné, si l'agent ne se plie pas aux normes et bien ça peut être une sanction... parce que ça devient la référence du métier on va dire.»

(Entretien avec le dirigeant de proximité Escale.)

Pour certains agents d'escale, le déploiement des normes de service a eu pour effet, non seulement de renforcer le pouvoir de l'encadrement, mais également d'introduire un rapport de servilité dans les relations agents-usagers, en faisant émerger la figure d'un «client roi», tout en les déshumanisant. L'agent d'escale était réduit, à leurs yeux, à un rôle d' «automate», de "pancarte», de «poteau indicateur $»$, ou encore de «porteur $»$, pour reprendre

(16) Selon Foucault (1975, p. 210), «Ce qui relève de la pénalité disciplinaire, c'est l'inobservation, tout ce qui est inadéquat à la règle, tout ce qui s'en éloigne, les écarts. Est pénalisable le domaine indéfini du non-conforme.» 
quelques-unes de leurs expressions favorites ${ }^{(17)}$. $\mathrm{Ce}$ refus explicite de respecter les règles dans certaines situations peut être interprété comme une «forme d'autoprotection» (DASSA, Maillard, 1996). «Ce à quoi l'organisation se heurte là [...] c'est à un certain sentiment de dépersonnalisation, d'intrusion dans la subjectivité, et dans une intimité jusque-là tenue à l'écart du champ et du contrat de travail. Quand la contrainte est ressentie par trop intrusive, l'individu résiste à une prise de pouvoir, de domination et de possession de soi et de son corps par l'organisation; la transgression correspond alors à une forme d'autoprotection» (Dassa, Maillard, 1996, p. 37) :

"Moi le mec qui arrive et qui me dit "Ici c'est Paris?" en me montrant le TGV, sans rien dire d'autre, là je me contente de dire "oui" [...]. Moi je n'aime pas que les clients me prennent pour un poteau indicateur! Il est hors de question que je dise "bon voyage" à un mec qui me prend pour une pancarte! [...] Qu'est-ce qu'on va nous demander maintenant? De cirer les chaussures des clients?»

«Une personne qui est en difficulté et qui nous le demande gentiment on l'aide. Mais c'est vrai qu'il y a des clients qui nous prennent pour des porteurs! Si c'est des gens polis, courtois, oui, mais sinon il y a des limites... »

(Entretien avec des agents d'accueil.)

Pour d'autres agents, ces «attitudes de service», parce qu'elles véhiculaient une représentation idéalisée de la relation de service, étaient inappropriées dans certaines situations, voire contre-productives. Elles ont donc été perçues davantage comme des contraintes que comme des ressources, d'autant plus qu'elles ont été une entrave à l'expression de leur personnalité dans le travail et qu'elles ont participé à la méconnaissance du travail réel des agents d'escale ${ }^{(18)}$. L'enquête de terrain a ainsi révélé que dans les situations perturbées (retards de train, grèves, etc.) au cours desquelles les agents d'escale devaient répondre simultanément, ou dans des temps très courts, à plusieurs demandes, ils devaient être capables de «se défaire» des clients les plus vindicatifs pour ne pas s'engager dans un «dialogue de sourds ». Pour ce faire, les agents adoptaient volontairement une attitude de fermeture, à rebours des «attitudes de service» prescrites. Mais ce faisant ils

(17) Selon Avarguez (2009), ce sentiment de déshumanisation de la relation, induit par la mise en place de normes de service rigides, doit être mis en relation avec le déni de la dimension sociale de la relation de service. L'auteur dresse ce constat à propos des conseillers à l'emploi de l'ANPE et souligne le «mouvement de chosification dans lequel ils sont enserrés et auquel ils prennent part» (AvArgueZ, 2009, p 58).

(18) La mise en œuvre des «attitudes de service» eut mécaniquement pour effet de gommer les spécificités propres au contexte dans lequel les relations de service se déployèrent. s'exposaient à des remontrances de la part de leur hiérarchie :

«Il y a des situations où on ne peut pas dire "Bonjour Monsieur” parce que ça n'a aucun sens. Je vais vous donner un exemple. Quelqu'un qui arrive complètement paniqué et qui nous interpelle c'est difficile de dire "Bonjour Monsieur" car ça n'a pas de sens! [...] Donc si on nous juge que là-dessus c'est un peu réducteur. On pourrait nous juger sur plein d'autres choses...»

À propos du respect des normes de service évaluées lors des entretiens annuels d'appréciation : «Moi je trouve que c'est bidon! Moi je trouve que je fais bien mon travail et lui ce qu'il me dit c'est que je ne mets pas ma casquette! On s'arrête sur des points de détail comme ça... parce que la norme c'est d'avoir la casquette sur la tête.»

«Si le train est en retard il faut se dire "il va desservir quoi?" Derrière ça il faut se dire "qu'est-ce qui va le plus $m$ 'arriver comme questions?" Donc le but c'est de tout de suite préparer les réponses parce que les gens ils vont être sur le quai et ils vont être pressés. Donc ils ne vont pas attendre le "Bonjour Monsieur", “Bonjour Madame”. Eux ce qu'ils veulent c'est prendre un train et finir leur voyage. »(19)

(Entretien avec des agents d'accueil.)

À l'inverse, ils avaient noué de véritables relations de proximité avec les personnes à mobilité réduite, notamment avec les jeunes, leurs «habitués». Ils adoptaient d'ailleurs avec eux et leurs proches une attitude teintée de familiarité (tutoiement, bises, échanges de menus services, etc.), au demeurant très éloignée des scripts langagiers qu'ils étaient censés respecter (voir encadré 5).

(19) Cet exemple illustre bien le décalage qui peut exister entre, d'un côté, la qualité prescrite (matérialisée ici par le strict respect d'une norme de politesse) et, de l'autre, la représentation que l'agent se fait de la situation et de ce que signifie délivrer un service de qualité (ici renseigner efficacement et rapidement les voyageurs). La norme de politesse vient, dans cet exemple, compliquer l'exercice du métier et introduit une dissonance au cour de l'activité. Comme le souligne très justement CLOT (2010, p. 112), c'est alors "le sens même de l'action en cours [qui] peut se perdre quand disparaît le rapport entre les buts auxquels il faut se plier, les résultats auxquels il faut s'astreindre et ce qui compte vraiment pour soi et pour les collègues dans la situation en question. Ce qui compte vraiment - et parfois de manière vitale dans les tâches de services - dessine d'autres buts possibles en termes de qualité que la qualité attendue des buts prescrits». 


\section{Encadré 5 \\ Extrait du journal de terrain du 8 juin 2007}

JM, agent d'escale, s'occupe de Sandrine, une jeune fille malvoyante qu'il s'agit d'acheminer jusqu'à son père. Arrivé devant les voies, JM bascule délicatement le fauteuil vers l'arrière et fait traverser Sandrine (S) en arrière.

$S$ : «Oh là. Ne me lâchez pas hein!»

JM : “Ah c'est qu'elle a peur la Sandrine! Ah t'inquiète pas va! On a l'habitude va.»

Le chercheur : "C'est un peu rock and roll! " Rire de Sandrine.

JM (arrivant dans le hall) : «Bon alors il est où le papa? Bon il doit être sur le parking en train de fumer sa cigarette. "

$\mathrm{E}$ (un autre agent) : «Normalement il devrait pas fumer avec ce qu'il a... »

JM (s'adressant à Sandrine) : "Tu feras gaffe Sandrine. Maintenant le dimanche c'est 18h05! Donc maintenant, le dimanche, le train qui descend sur Lyon, tu diras à ton papa que c'est 18 h05 et non pas $18 h 15$ ! »

$S:$ : 18h05. »

JM : «Voilà!»

JM : «Les horaires vont changer dimanche. »

JM (en aparté, commentant la situation au chercheur) : «Bon de toute façon je vais le dire au papa. II vaut mieux... "

JM (saluant le père de Sandrine) : «Bonjour. Alors vous ferez attention maintenant le dimanche c'est 18h05. "

Le père : «Ah bon!»

JM : «Vous avez déjà vos billets?»

Le papa : «Non. Mais c'est toujours 18 h05 maintenant? »

JM : “C'est 18 h05 au lieu de 18h15. Non mais je vous le dis parce que vous avez tellement l'habitude de venir à $18 h 15 . . . »$

Au final, les contrôles de conformité réalisés par les enquêteurs client mystère de la société prestataire ont totalement occulté le travail réel des agents d'escale, les difficultés et les tensions inhérentes à leur activité, et l'étendue de leurs compétences. Par exemple, les agents étaient régulièrement amenés à gérer des situations dans lesquelles des contraintes de différentes natures étaient enchevêtrées. Soumis à des injonctions contradictoires, ils devaient alors établir des priorités et procéder à des arbitrages. Ainsi, ceux qui assuraient les départs de train en gare devaient très fréquemment arbitrer entre les exigences liées à la sécurité et celles liées à la relation de service. Si les plus expérimentés étaient parfaitement capables d'articuler différents registres d'action (voir encadré 6), il n'en allait pas de même pour les autres.

\section{Encadré 6 \\ Le "bras de fer"}

L'agent vient de procéder au départ du train. Deux jeunes voyageurs arrivent en trombe sur le quai et se précipitent vers le train.

L'agent s'interpose en adoptant une attitude de grande fermeté afin de faire respecter les procédures de sécurité : “C'est terminé! On ne monte pas!» L'agent fait barrage avec son corps et fixe les deux jeunes droit dans les yeux. Ils obtempèrent.

L'agent, calmement, adoptant une «attitude de service» : "Alors le prochain pour Belfort c'est $18 h 34$ ».

L'agent, commentant la situation après coup : "C'est vrai que quand je dis qu'on ne monte pas on ne monte pas! Quand j'ai dit non c'est non! [...] Ça c'est un apprentissage qui se fait sur le terrain. Le "bras de fer" c'est sur le terrain".

Ces tensions étaient également fortement ressenties par l'encadrement intermédiaire souvent sollicité par les agents pour arbitrer entre des exigences difficilement conciliables, mais aussi parce qu'il devait régulièrement rendre des comptes à une pluralité d'acteurs situés au-dessus de lui. Le dirigeant de proximité Escale explique ainsi :

«Normalement, quand le train part, une fois que le départ est donné, l'agent est censé observer tout le défilé du train de manière à détecter une anomalie. Et c'est typiquement là où ça peut être difficile car le client il voit que l'agent a donné le départ du train et donc il pose sa question. Alors si c'est une question pour laquelle il faut trente secondes pour répondre, $s$ 'il se fait "extraire", l'agent $n$ 'aura pas fait sa mission de sécurité. [...] Alors je ne prioriserais pas entre les deux, car suivant l'interlocuteur, je tombe souvent sur des intégristes de la sécurité qui vont me dire qu'il n'y a que la sécurité! Je tombe aussi sur d'autres intégristes qui me disent l'inverse, à savoir que le client est prioritaire et que tout le reste est accessoire! \»

Les normes de service mises en place dans le cadre de la démarche Gares en mouvement ont, en définitive, contribué au déni du travail des agents d'escale de la SNCF. Elles ont notamment eu pour effet d'occulter la dimension sociale de la relation de service (la réalité du travail), de masquer les contradictions et les difficultés rencontrées par les agents sur le terrain (le «réel du travail»), de même que les compétences requises pour traiter efficacement certaines situations délicates (le travail réel). 


\section{Quand les normes deviennent une fin en soi}

Dans les deux cas étudiés, ces normes ont finalement été perçues par la majorité du personnel en front office comme un obstacle à la réalisation d'un travail de qualité. La qualité du service rendu ne peut effectivement pas être appréhendée sur la seule base du respect des normes ${ }^{(20)}$. Assurer une simple présence en gare pour «humaniser» les lieux, respecter les normes vestimentaires définies par la direction, ou encore s'en tenir au strict respect des formules de politesse contenues dans les scripts langagiers, ne la garantissent en rien. Au mieux peuvent-elles y contribuer. Selon de Gaulejac (2005), les normes préétablies ne permettent donc pas réellement de mesurer la qualité du service, «l'agent et le destinataire étant les mieux placés pour évaluer la relation de service», c'est-à-dire pour «formuler un jugement sur le service rendu» (p. 124). Les attentes des clients sur la qualité du service peuvent en effet ne pas correspondre aux «promesses» ou aux «engagements» pris par l'entreprise dans le cadre des procédures qualité et ce, malgré les dispositifs d'évaluation et de contrôle mis en place (comme les enquêtes client mystère) ${ }^{(21)}$. Par ailleurs, les agents en front office ont généralement leur propre idée sur ce que doit être un travail de qualité, idée qui peut donc s'avérer assez éloignée des critères de qualité véhiculés par les normes de service. Le bien-fondé des normes peut par conséquent être contesté, aussi bien par le client final que par l'agent chargé de délivrer le service.

Cependant, les normes que nous avons décrites se sont révélées très pesantes dans les deux cas étudiés. Si, au départ, elles étaient censées être un moyen d'atteindre l'objectif légitime d'améliorer la qualité du service rendu au client, leur respect est devenu, à l'arrivée, une fin en soi. Ce constat rejoint, à bien des égards, celui dressé par Delphine Mercier (2001) à propos de la mise en place d'une démarche de certification dans les centres de tri de La Poste. Ce type de situation a pour effet de créer une confusion dans les esprits, les dirigeants et l'encadrement ayant alors tendance à confondre qualité prescrite et qualité réelle. En amont, le piège réside dans le fait de mesurer exclusivement la qualité des services rendus au regard des seules normes prescrites et de s'en tenir là, en oubliant au passage l'évaluation faite par le destinataire du service mais également par l'agent en contact direct avec le client. Dans les cas que nous avons présentés,

(20) Signalons que pour certains auteurs, dans la perspective dessinée par la psychodynamique du travail, «il est [même] impossible d'atteindre la qualité en respectant scrupuleusement les prescriptions » (GANEM, 2011, p. 131).

(21) Ces dispositifs ne permettent finalement que d'évaluer les procédures de délivrance du service au regard des standards préalablement définis. cette situation nous semble au fondement du déni du travail des agents en front office de la $\mathrm{SNCF}^{(22)}$. Mais comment expliquer cette focalisation des dirigeants et de l'encadrement sur les normes prescrites au détriment du travail réalisé par les agents au contact de la clientèle?

Il faut revenir brièvement sur le contexte dans lequel ces deux démarches ont été mises en œuvre pour répondre à cette question. Sous le prétexte d'amélioration de la qualité des services rendus, leur lancement s'est accompagné de l'introduction de relations de type client-fournisseur (voir figures 1 et 2 présentées plus haut) entre les donneurs d'ordre (23) et les directions d'activité (24), mais également entre ces dernières et les unités opérationnelles. La contractualisation s'est effectuée sur la base de standards de service, édictés par les producteurs de normes que sont les donneurs d'ordre et les directions d'activité. Ces derniers avaient en effet décidé de standardiser le service en définissant des normes et des indicateurs clairs, "objectifs», atteignables, et surtout mesurables. Dès lors, il était possible de mesurer le degré de conformité des gares par rapport à des standards préalablement définis ${ }^{(25)}$. Ce faisant, le respect des normes de service est devenu la priorité pour l'ensemble des acteurs concernés et ces normes se sont révélées être une véritable «technologie invisible» (BERRY, 1983) : pour les donneurs d'ordre qui entendaient rémunérer les directions d'activité en fonction du degré de mise en conformité des gares; pour les directions d'activité qui s'étaient engagées à honorer leurs «promesses» et qui devaient présenter un compte de résultats excédentaire (voir encadré 7).

(22) Valérie BoussARD (2003), reprenant le travail de recherche de Delphine Mercier réalisé au sein de La Poste, parle, quant à elle, d' «effet d'aveuglement» pour rendre compte du processus d'occultation du travail réellement effectué par les agents des centres de tri pour réaliser un travail de qualité.

(23) Le Syndicat des transports en Île-de-France dans un cas. Les branches transport public et voyageurs France Europe dans l'autre.

(24) La Direction Île-de-France dans un cas. La Direction des gares et de l'escale dans l'autre.

(25) De Gaulejac (2005, p. 71) souligne, à propos des outils de mesure de la qualité, qu'ils «donnent l'illusion que la réalité peut être comprise et maîtrisée à condition de pouvoir la mesurer. Pour ce faire, on découpe cette réalité en particules que l'on voudrait élémentaires, auxquelles on affecte un coefficient. Une fois le découpage et le chiffrage effectués, tous les calculs sont possibles». C'est exactement ce que nous avons observé dans le cas des deux démarches qualité décrites dans cet article. Cette situation est, selon l'auteur, le symptôme d'une «maladie de la mesure» désignée sous le terme de «quantophrénie» : «la quantophrénie désigne une pathologie qui consiste à vouloir traduire systématiquement les phénomènes sociaux et humains en langage mathématique» (p. 70). 


\section{Encadré 7}

\section{La prégnance des normes de service et des mécanismes de contractualisation}

Dans le cas du projet Transilien et humanisation des gares, le Syndicat des transports en Île-deFrance (donneur d'ordre) évaluait et rémunérait la Direction île-de-France de la SNCF (direction d'activité) - via un système de bonus-malus - en fonction des engagements pris par cette dernière, au premier rang desquels figurait la présence en gare d'au moins deux agents de la SNCF jusqu'au passage du dernier train. Une gare qui ne respectait pas ce standard ne pouvait d'ailleurs pas obtenir le label Transilien et la SNCF se voyait alors attribuer un malus. On comprend donc pourquoi, lors du lancement du Transilien et de l'humanisation des gares, la priorité de la Direction Île-de-France fut la mise en place des équipes d'humanisation. Peu importe finalement ce que faisaient les agents en gare, l'essentiel était de tenir ses engagements à court terme et d'obtenir le précieux label.

Dans le cas de la démarche Gares en mouvement, les branches transport public et voyageurs France Europe (donneurs d'ordre) évaluaient et rémunéraient les services rendus par la Direction des gares et de l'escale (direction d'activité) en fonction du respect des normes de service, au premier rang desquelles figuraient les «attitudes de service». Les mesures réalisées régulièrement par les enquêteurs client mystère permettaient, in fine, de calculer un indice de conformité des comportements des agents d'escale aux «attitudes de service» et d'attribuer à chaque gare une note.

Le respect des normes devint également une priorité pour l'encadrement principalement évalué en fonction de sa capacité à les faire appliquer par le personnel. Ainsi, lors du lancement du Transilien et de l'humanisation des gares, la priorité de la Direction de l'Établissement de Melun fut de trouver des volontaires parmi les agents d'ambiance et les agents commerciaux pour assurer l'ouverture des gares jusqu'au passage du dernier train et constituer des binômes agent d'ambiance/ agent commercial sur chacune des gares. Dans le second cas, la Direction des gares et de l'escale impulsa des challenges durant les «temps forts» qui correspondaient à des périodes durant lesquelles les contrôles de conformité étaient renforcés par la multiplication des enquêtes client mystère. Les notations et les classements qui en résultaient, ainsi que les audits réalisés deux fois par an par cette direction, eurent pour effet de créer une pression environnementale forte : autant de procédures qui ont permis in fine à la Direction des gares et de l'escale d'exercer son pouvoir sur les unités opérationnelles et l'encadrement intermédiaire. Les «attitudes de service» étaient d'ailleurs d'autant plus prégnantes que l'encadrement intermédiaire était évalué sur sa capacité à faire respecter ces normes. Le dirigeant de l'unité opérationnelle production voyageurs, supérieur hiérarchique du dirigeant de proximité Escale indique ainsi :

«Ce n'est plus la "SNCF de papa”! [...] Les transporteurs [les branches transport public et voyageurs France Europe] expriment un certain nombre d'attentes, dont des attentes économiques très fortes. Ils payent un service à l'Escale et donc ils veulent avoir le meilleur service au moindre coût. C'est une logique incontournable! »

Le dirigeant de proximité Escale ajoute : «Les clients mystères c'est la Bible! [...] C'est vrai que l'aspect purement normatif est un peu contraignant parce que quand on a une enquête qui n'est pas bonne en termes de pourcentages, inévitablement ça nous retombe dessus on va dire et il faut rendre des comptes. »

Dans les deux cas étudiés, le déploiement à grande échelle d'une politique de qualité, par un processus de certification des gares et la mise en place de toute une panoplie d'outils (système de bonus-malus, «attitudes de service», enquêtes client mystère, etc.), a permis aux dirigeants et à l'encadrement de disposer de «références communes» (BoIGNe et al., 1986) et d'indicateurs simples, tels que le nombre de gares certifiées ou labellisées ${ }^{(26)}$, le respect des standards de service, l'indice de conformité des comportements des agents aux «attitudes de service», etc. Ces indicateurs - bien que rudimentaires, car ne rendant pas compte de la complexité de l'activité des agents - permirent aux différents acteurs de contractualiser leurs relations, de dresser périodiquement des bilans et des évaluations, de comparer les gares entre elles, d'imputer des responsabilités, de distribuer des récompenses et des sanctions, de déterminer des objectifs à atteindre, d'orienter les conduites, bref de structurer la réalité en la simplifiant et de stabiliser les rapports sociaux (BERRY, 1983)(27). $\mathrm{Au}$ nom du client final, la diffusion de normes de service, d'outils et d'indicateurs de qualité dans l'organisation a eu pour effet d'enraciner le pouvoir des producteurs de normes, donneurs d'ordre et directions d'activité, de conforter les relations de pouvoir entre les différents acteurs et de légitimer chacun dans son rôle. Cependant, dans le même temps, elle a favorisé le déni du travail des agents en front office.

(26) Dans le cas de l'humanisation des gares, rappelons que l'enjeu pour les dirigeants était d'obtenir auprès du Syndicat des transports en Île-de-France le précieux label Transilien SNCF. Dans le second cas, l'enjeu pour la Direction des gares et de l'escale était de faire certifier un maximum de gares sous son contrôle et, pour l'encadrement intermédiaire de la gare que nous avons pris pour exemple, de conserver sa certification (puisqu'elle avait été certifiée NF Service par l'Agence française de normalisation [AFNOR]).

(27) Comme le soulignent Dassa et Maillard (1996, p. 35), «la prescription porte en elle, non seulement des énoncés d'actions instrumentalisées, mais aussi de manière cachée, secrète, inconsciente, "naturalisée", des rapports sociaux de dépendance et de contrôle. La procédure, au-delà du discours, continue de séparer prescripteurs et réalisateurs ». 
Les mécanismes organisationnels (du processus de contractualisation aux «défaillances» de la prescription en passant par la priorité accordée au respect des normes par les dirigeants et l'encadrement) que nous avons décrits à propos de deux démarches qualité déployées à la SNCF dans le domaine de la relation de service ont contribué au déni du travail des agents en front office. Le contexte social dans lequel s'exercent les relations de service (la réalité du travail), les difficultés et les obstacles rencontrés par les agents dans leur activité (le «réel du travail»), mais également les pratiques et les compétences professionnelles qu'ils ont développées pour conjurer le réel (le travail réel), furent totalement occultés et firent l'objet d'un déni de la part des producteurs de normes. En effet, les normes de service que nous avons analysées eurent non seulement pour conséquence de gommer les aspérités du réel - c'est-à-dire les particularismes locaux, la singularité des situations gérées par les agents en front office, la nature et la complexité des problèmes rencontrés, etc. - mais permirent également aux producteurs de normes de congédier et de chasser un réel très, voire trop encombrant.

Nous pouvons alors nous demander si, du côté des producteurs de normes, nous n'avons pas affaire à «une rupture collective du lien avec le réel» (Dejours, 2006, p. 131), autrement dit à ce que François Sigaut (2004) a théorisé sous le terme d'«aliénation culturelle». Selon lui, cette aliénation se produit quand l'expérience du réel d'un individu ou d'un collectif est déniée par un groupe social pour qui cette expérience est profondément dérangeante. S'ensuit alors « une coupure [qui] décrit la situation d'un groupe ou d'un sous-groupe social dans lequel l'impératif de solidarité entre ses membres [...] est placé au-dessus de la prise en considération du réel» (Sigaut, 2004, p. 121 et 122).

\section{Bibliographie}

Avarguez S. (2009), «Des "réformes venues d'en haut" aux "réformes venues d'en bas". Une approche sociologique du travail vécu des conseillers à l'emploi de l'ANPE entre insatisfaction et souffrance», Pyramides, $\mathrm{n}^{\circ} 17$, pp. 53-68.

Bardou H., Vacheron-Trystram M.-N., Cheref S. (2006), «Le déni en psychiatrie», Annales médicopsychologiques, vol. 164, $\mathrm{n}^{\circ}$ 2, pp. 99-107.

Batisse F. (2002), «L’Escale», Revue générale des chemins de fer, $\mathrm{n}^{\circ} 108$, pp. 31-32.

BerRY M. (1983), Une technologie invisible? L'impact des instruments de gestion sur l'évolution des systèmes humains, Paris, Centre de recherche en gestion de l'école polytechnique.

Boigne J.-M., Moisdon J.-C., Tonneau D. (1986), «Gérer ou comprendre? Perplexités à propos d'une intervention en milieu hospitalier», Gérer et comprendre, $\mathrm{n}^{\circ}$ 5, pp. 78-86.

Borzeix A., Collard D. (1999), «La gestion des gares de banlieue est-elle une compétence?», Éducation permanente, $\mathrm{n}^{\circ} 141$, pp. 83-96.

Borzeix A., Collard D., Raulet-Croset N., Teulier R. (2008), Les compétences implicites dans la relation de service à la SNCF, PREG-CRG École Polytechnique, rapport de recherche réalisé pour le compte de la SNCF.

BousSARD V. (2003), «Dispositifs de gestion et simulacres de contrôle", in Boussard V., Maugeri S., Du politique dans les organisations. Sociologies des dispositifs de gestion, Paris, l'Harmattan, coll. «Logiques sociales. Série Sociologie de la gestion», pp. 173-191.
CAUCHON C. (1998), «Le modèle public de modernisation à la recherche d'une nouvelle régulation : un processus engagé mais non abouti à la SNCF», Politiques et management public, vol. 16, $\mathrm{n}^{\circ}$ 4, pp. 19-39.

Clot Y. (1995), Le travail sans l'homme. Pour une psychologie des milieux de travail et de vie, Paris, la Découverte, coll. «Textes à l'appui».

CLот Y. (1999), La fonction psychologique du travail, Paris, Presses universitaires de France, coll. «Le Travail humain».

Clot Y., Faïta D. (2000), «Genres et styles en analyse du travail. Concepts et méthodes», Travailler, $\mathrm{n}^{\circ} 4$, pp. 7-42.

CLот Y. (2010), Le travail à cour, pour en finir avec les risques psychosociaux, Paris, la Découverte, coll. «Cahiers libres».

Collard D. (2001), «Activités et emplois de médiation sociale en gare. Quelles pratiques et quelles compétences?», Informations sociales, $\mathrm{n}^{\circ}$ 92, pp. 32-41.

Collard D. (2002), La médiation : une compétence ingérable? Le cas des emplois-jeunes de la SNCF, Thèse de doctorat de l'École polytechnique, Paris.

Collard D. (2003), "Analyser les compétences des médiateurs dans les gares de banlieue», Travail et emploi, $\mathrm{n}^{\circ} 94$, pp. 37-43.

Collard D. (2010), «De la stratégie orientée client aux "enquêtes client mystère". Les "enquêtes client mystère" à la SNCF : une "fiction" aux effets pourtant bien réels !», Gérer et comprendre, ${ }^{\circ} 102$, pp. 36-46. 
Dassa S., Maillard D. (1996), «Exigences de qualité et nouvelles formes d'aliénation", Actes de la recherche en sciences sociales, $\mathrm{n}^{\circ} 115$, pp. 27-37.

Dejours C. (1995), Le facteur humain, Paris, Presses universitaires de France, coll. «Que sais-je?».

Dejours C. (1998), Souffrance en France. La banalisation de l'injustice sociale, Paris, Éditions du Seuil, coll. «L'histoire immédiate».

Dejours C. (2006), «Aliénation et clinique du travail», Actuel Marx, vol. 1, n 39, pp. 123-144.

DivaY S. (2004), «Quand les compétences ethnicisées facilitent l'insertion professionnelle», Hommes et migrations, $\mathrm{n}^{\circ} 1249$, pp. 87-96.

Dujarier M.-A. (2004), L'idéal au travail dans les services de masse, Thèse de sociologie, Université de Paris VII Denis Diderot.

FAVIN-LeVeque J.-C. (2005), «La politique de services de la direction des gares et de l'escale», Revue générale des chemins de fer, $\mathrm{n}^{\circ} 135$, pp. 19-30.

Foucault M. (1975), Surveiller et punir. Naissance de la prison, Paris, Gallimard, coll. «Bibliothèque des histoires ».

Freud S. (1909), «Analyse d'une phobie chez un garçon de 5 ans (Le Petit Hans)», in Freud S., Cinq psychanalyses, Paris, Presses universitaires de France, 1954, pp. 93-198.

Ganem V. (2011), «Seul le travail rentable est évalué», Travailler, $\mathrm{n}^{\circ} 25$, pp. 129-143.

Gaulejac V. (de) (2004), La société malade de la gestion, Paris, Éditions du Seuil, coll. «Économie humaine».

Gollac M., Castel M.-J., Jавot F., Presseq P. (2006), «Du déni à la banalisation», Note de recherche : Sur la souffrance mentale au travail, Actes de la recherche en sciences sociales, $\mathrm{n}^{\circ} 163$, pp. 39-45.

Grangaud N. (2001), Déni de grossesse : description clinique et essai de compréhension psychopathologique, Thèse de médecine, Université de Paris VII.

Grimand A. (2004), «L'évaluation des compétences : paradoxes et faux semblants d'une instrumentation », $X V^{e}$ Congrès Annuel de l'AGRH, Montréal.
Guelaud C. (2002), «La SNCF : une stratégie de croissance, un imaginaire du déclin », in Tixier P.-E. (dir.), Du monopole au marché. Les stratégies de modernisation des entreprises publiques, Paris, la Découverte, coll. «Textes à l'appui», pp. 126-146.

LADA E. (2003), «Agents d'ambiance et de médiation : une construction sociale sexuée et ethnicisée en devenir», in Divay S. (ed.), «Regards croisés sur les emploisjeunes », Documents du Céreq, n 173, pp. 131-141.

Lhuilier D. (2006), «Cliniques du travail», Nouvelle revue de psychosociologie, vol. 1, $\mathrm{n}^{\circ} 1$, pp. 179-193.

Lhuilier D. (2009), «Travail, management et santé psychique», Connexions, n 91, pp. 85-101.

Mercier D. (2001), « Heurts et malheurs de la certification dans les centres de tri de la poste», in Maugeri S. (dir.), Délit de gestion, Paris, La Dispute, pp. 31-50.

Perilleux T. (2005), «Le déni de l'évaluation», Travailler, $\mathrm{n}^{\circ} 13$, pp. 113-134.

Petitclerc J.-M. (2002), Pratiquer la médiation sociale, Paris, Dunod.

Pouvelle P. (1998), «Les services en gare et la satisfaction de la clientèle : les différents métiers d'escale dans les gares ", Revue générale des chemins de fer, $\mathrm{n}^{\circ} 4$, pp. 15-21.

Royer C., Lautier R., Laurent R., Ostalier S. (2004), «Pourquoi la mise en œuvre des escales et les certifications de service», Revue générale des chemins de fer, $\mathrm{n}^{\circ} 129$, pp. 25-31.

Sigaut F. (2004), «Folie, réel et technologie. À propos de Philippe Bernardet, Les Dossiers noirs de l'internement psychiatrique, Paris, Fayard, $1989 »$, Travailler, $\mathrm{n}^{\circ}$ 12, pp. 117-130.

Williot J.-P. (2005), «Un nouveau chantier d'archives orales : l'histoire des décisions stratégiques de la SNCF et de leur application depuis les années 1970 », Revue d'histoire des chemins de fer, $\mathrm{n}^{\circ} 31, \mathrm{pp}$. 29-61. 